ARTICLE OPEN

Check for updates

\title{
7,8-dihydroxyflavone ameliorates motor deficits via regulating autophagy in MPTP-induced mouse model of Parkinson's disease
}

\author{
Li Zuo ${ }^{1}$, Chunfang Dai ${ }^{1}$, Lilin $\mathrm{Yi}^{1}$ and Zhifang Dong ${ }^{1}{ }^{1 凶}$ \\ (c) The Author(s) 2021
}

Parkinson's disease (PD) is a neurodegenerative disease characterized by the loss of dopaminergic neurons in the substantia nigra and diminished dopamine content in the striatum. Recent reports show that 7,8-dihydroxyflavone (DHF), a TrkB agonist, attenuates the a-synuclein deposition and ameliorates motor deficits. However, the underlying mechanism is unclear. In this study, we investigated whether autophagy is involved in the clearance of a-synuclein and the signaling pathway through which DHF exerts therapeutic effects. We found that the administration of DHF $(5 \mathrm{mg} / \mathrm{kg} /$ day, i.p.) prevented the loss of dopaminergic neurons and improved motor functions in the 1-methyl-4-phenyl-1,2,3,6-tetrahydropyridine (MPTP) mouse model of PD, whereas these protective effects of DHF were completely blocked by autophagy inhibitor chloroquine (CQ). Further in vitro studies showed that autophagy was inhibited in N2A cells treated with 1-methyl-4-phenylpyridinium $\left(\mathrm{MPP}^{+}\right)$, as reflected by a significant decrease in the expressions of autophagy marker proteins (Beclin1 and LC3II) and an increase in the expression of autophagic flux marker p62. DHF restored the impaired autophagy to control level in $\mathrm{MPP}^{+}$-treated N2A cells by inhibiting the ERK-LKB1-AMPK signaling pathway. Taken together, these results demonstrate that DHF exerts therapeutic effects in MPTP/MPP ${ }^{+}$-induced neurotoxicity by inhibiting the ERK-LKB1-AMPK signaling pathway and subsequently improving impaired autophagy.

Cell Death Discovery (2021)7:254 ; https://doi.org/10.1038/s41420-021-00643-5

\section{INTRODUCTION}

Parkinson's disease (PD) is a neurodegenerative disorder with high incidence that only second to Alzheimer's disease. The main pathological changes of PD are the loss of dopaminergic neurons and deposition of intraneuronal inclusions known as Lewy bodies [1]. Even though the pathogenesis of PD has not been fully clarified, accumulating studies have shown that various intracellular processes are involved in it, including misfolding and aggregation of a-synuclein, endoplasmic reticulum stress, mitochondrial dysfunction, dysregulated calcium homeostasis, and dysfunctional autophagy $[2,3]$.

Especially, autophagy has been drawn a lot of attention in recent years. It has been reported that impaired autophagy exists in the brains of both PD patients and PD animal models [4-6]. Animal models with disrupted autophagy-lysosome system caused by deletion of essential autophagy gene are more likely to show PD-like pathology, which further confirmed that autophagy plays a neuroprotective role during the pathogenesis of PD $[6,7]$. As a crucial determinant of neurotoxicity, the level of a-synuclein is confirmed to be closely related to autophagy in recent studies [8]. The degradation of a-synuclein was majorly dependent on ALP (autolysosome pathway) and UPS (ubiquitinproteasome pathway) [9]. It has been reported that the degradation capacity of UPS is reduced in aged animals [10], resulting in a-synuclein aggregation and consequently contributing to PD pathogenesis. However, autophagy may compensate for the decreased degradation mediated by UPS $[9,10]$. Therefore, promoting autophagy through pharmacological or other methods to increase the degradation of a-synuclein may be a potential therapeutic target for PD [11].

Brain-derived neurotrophic factor (BDNF) can regulate a variety of biological processes in human body, especially in neurological system. It binds to the TrkB receptor and triggers dimerization and autophosphorylation of tyrosine residues in the intracellular domain, resulting in activation of signaling pathways including mitogen-activated protein kinase (MAPK), phosphatidylinositol 3-kinase (PI3K) and phospholipase- $\gamma$ (PLC- $\gamma$ ) [12]. BDNF displays therapeutic effects in numerous neurological, mental and metabolic disorders $[13,14]$. However, the use of recombinant BDNF has turned out to be disappointingly useless in clinical trials, probably due to poor drug delivery to the brain, short half-life period, and other factors that restrict its clinical use $[15,16]$. It is exciting that 7,8-dihydroxyflavone (DHF) is screened from thousands of compounds, which is orally bioavailable and easily penetrates the brain-blood barrier (BBB) to exert its neurotrophic effects in the central nervous system (CNS) $[12,17,18]$.

\footnotetext{
${ }^{1}$ Pediatric Research Institute, Ministry of Education Key Laboratory of Child Development and Disorders, National Clinical Research Center for Child Health and Disorders, China International Science and Technology Cooperation Base of Child Development and Critical Disorders, Chongqing Key Laboratory of Translational Medical Research in Cognitive Development and Learning and Memory Disorders, Children's Hospital of Chongqing Medical University, Chongqing 400014, China. ${ }^{凶}$ email: zfdong@cqmu.edu.cn Edited by Dr. Richard Killick
}

Received: 9 March 2021 Revised: 26 August 2021 Accepted: 7 September 2021

Published online: 20 September 2021 
Importantly, compared with BDNF, DHF has better clinical application characteristics. For instance, BDNF activated TrkB receptor and elicited receptor ubiquitination and degradation, while DHF does not induce TrkB ubiquitination or degradation [19]. In addition, DHF specifically acts on TrkB receptor, but BDNF also binds to p75NTR that could mediate the cell death pathway [20], apart from the TrkB receptor. Several studies have shown that treatment with DHF prevents a-synuclein accumulation and loss of dopaminergic neurons as well as ameliorating motor deficits in the MPTP-induced PD mouse model [12, 21, 22]. Consistent with that, our recent study shows that DHF treatment suppresses $a-$ synuclein accumulation and reduces oxidative stress, and subsequently blocks dopaminergic neuron loss in the substantia nigra (SN) and striatum [23]. However, the mechanism of DHF reducing the a-synuclein accumulation remains largely unclear. As forementioned, the accumulation of a-synuclein was closely connected with impaired autophagy. Previous studies have shown that BDNF-TrkB promotes autophagy flux [24, 25]. In addition, DHF binds to TrkB receptor and regulates the downstream signaling cascade including PI3K-AKT, MAPK, which play a role in the regulation of autophagy $[26,27]$. We, therefore, supposed that DHF may reduce a-synuclein aggregation via promoting autophagy. In the present study, we investigated this hypothesis by using a combination of biochemical, pharmacological, and behavioral assessments in PD models of both in vivo and in vitro.

\section{RESULTS}

Autophagy inhibitor chloroquine abrogates the protective effects of DHF on motor deficits in MPTP-induced mouse model of PD

Alterations in the function of autophagy to degrade misfolded and aggregated proteins or to eliminate damaged mitochondria are being recognized as an important factor in the pathogenesis of PD $[28,29]$. Our recent study has shown that DHF treatment ameliorates MPTP-induced motor deficits and reduces the loss of dopaminergic neurons in mice [23]. To determine whether autophagy is involved in these effects, we introduced autophagy inhibitor chloroquine in the present study. Three behavioral tests were conducted: rotarod test, pole test, and wire suspension test.

In rotarod test, mice in MPTP-treated group spent significantly less time on the rod compared with controls (CTR), and DHF treatment $(5 \mathrm{mg} / \mathrm{kg}$, i.p.) prolonged the latency on the rod. However, the beneficial effect of DHF was diminished by autophagy inhibitor chloroquine (CQ) treatment, as reflected by the latency decreasing to the same level of MPTP (CTR: $n=15$; MPTP: $n=19 ;$ MPTP + DHF: $n=19 ; \mathrm{MPTP}+\mathrm{DHF}+\mathrm{CQ}: n=22$; MPTP + CQ: $n=15$; Fig. 1a). In pole test, the mice in MPTP group spent much more time on descending to the floor compared with CTR, and DHF treatment significantly shortened the descending time. Similar to the findings in rotarod test, chloroquine markedly increased the descending time, indicating it abrogated the protective effect of DHF (CTR: $n=15,8.9 \pm 0.5$ s; MPTP: $n=19$, $12.9 \pm 1.0 \mathrm{~s}, P<0.05$ vs. CTR; MPTP + DHF: $n=19,8.8 \pm 0.5 \mathrm{~s}, P>$ 0.05 vs. CTR, $P<0.05$ vs. MPTP; MPTP + DHF + CQ: $n=22,14.8 \pm$ $1.3 \mathrm{~s}, P<0.001$ vs. MPTP + DHF; MPTP + CQ: $n=15,18.8 \pm 2.2 \mathrm{~s}$, $P>0.05$ vs. MPTP; Fig. 1b). Finally, in wire suspension test, DHF treatment shortened the time to reach the platform in MPTPtreated mice, while chloroquine treatment made it back to MPTP group level (CTR: $n=15,16.5 \pm 0.8 \mathrm{~s}$; MPTP: $n=19,21.4 \pm 1.4 \mathrm{~s}$, $P<0.01$ vs. CTR; MPTP + DHF: $n=19,16.1 \pm 0.6 \mathrm{~s}, P>0.05$ vs. CTR, $P<0.05$ vs. MPTP; MPTP + DHF + CQ: $n=22,22.4 \pm 0.9 \mathrm{~s}, P<0.001$ vs. MPTP + DHF; MPTP + CQ: $n=15,21.8 \pm 0.7 \mathrm{~s}, P>0.05$ vs. MPTP; Fig. 1c). Notably, chloroquine alone had no influence on motor functions in MPTP-treated mice (Fig. 1a-c). Together, these results suggest that DHF rescued the motor deficits in MPTPinduced PD mice, while chloroquine prevents the protective effects of DHF.
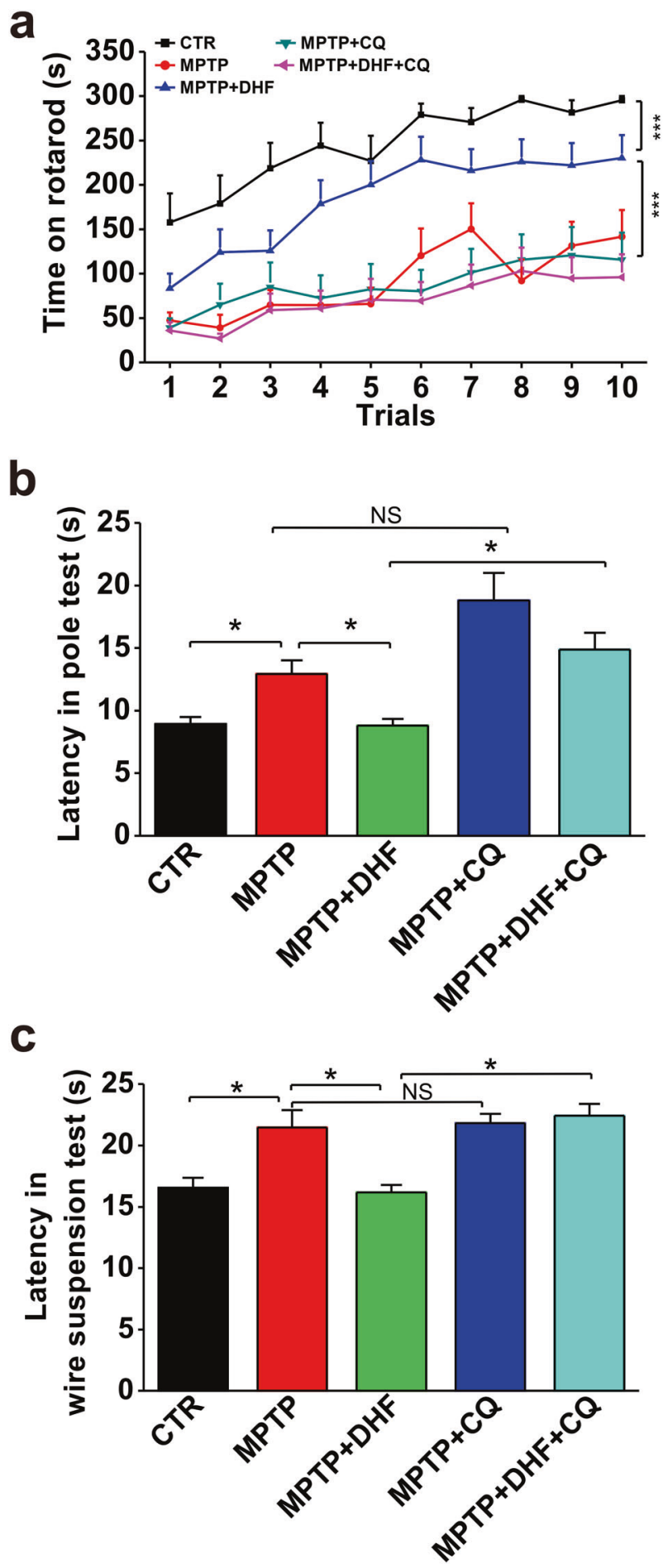

Fig. 1 Chloroquine (CQ) prevents the protective effects of DHF on motor deficits in MPTP-induced mouse model of PD. a Latency to fall off the rod in the rotarod test was significantly reduced by MPTP administration, and DHF treatment restored the latency to control level. CQ abolished the recovery of DHF in MPTP-induced PD mouse. b Latency to descend in the pole test was markedly increased by MPTP administration and DHF treatment restored the latency to control level. CQ abrogated the recovery of DHF in MPTP-induced PD mouse. c Latency to reach the platform in the wire suspension test was significantly increased by MPTP administration, and DHF treatment restored the latency to control level while CQ abolished the recovery of DHF in MPTP-induced PD. Data are expressed as mean \pm SEM. ${ }^{*} P<0.05,{ }^{* * *} P<0.001$. 
Chloroquine prevents the effects of DHF on the restoration of TH and the decrease of a-synuclein accumulation

The accumulation of a-synuclein and the lack of tyrosine hydroxylase $(\mathrm{TH})$, a rate-limiting enzyme during biosynthesis of L-dihydroxyphenylalanine (L-DOPA), are thought to contribute to PD progression [30]. Therefore, we next examined the a-synuclein and TH expression in the striatum and midbrain in MPTP-induced PD model mice with or without chloroquine treatment. The results showed that the MPTP administration significantly increased asynuclein accumulation and decreased TH expression in both the striatum (a-synuclein: $\mathrm{n}=5$ in each group; MPTP: $1.6 \pm 0.2$ relative to CTR, $P<0.05$ vs. CTR; TH: $n=5$ in each group; MPTP: $0.54 \pm 0.03$ relative to $C T R, P<0.01$ vs. CTR; Fig. $2 a-c)$ and midbrain (asynuclein: $n=5$ in each group; MPTP: $1.52 \pm 0.06$ relative to CTR, $P<0.01$ vs. CTR; TH: $n=5$ in each group; MPTP: $0.56 \pm 0.04$ relative to CTR, $P<0.01$ vs. CTR; Fig. $2 \mathrm{e}-\mathrm{g}$ ), compared with those in control group. Consistent with our recent report [23], DHF treatment restored a-synuclein accumulation and $\mathrm{TH}$ expression to control levels (a-synuclein: MPTP + DHF: $1.03 \pm 0.06$ relative to CTR, $P<$ 0.05 vs. MPTP; TH: MPTP + DHF: $1.57 \pm 0.25$ relative to CTR, $P<$ 0.05 vs. MPTP Fig. 2a-c, e-g). However, the effects of DHF on increasing $\mathrm{TH}$ expression and reducing a-synuclein accumulation were completely prevented by chloroquine treatment (a-synuclein: $\mathrm{MPTP}+\mathrm{DHF}+\mathrm{CQ}: 1.58 \pm 0.22$ relative to $\mathrm{CTR}, P<0.05$ vs. MPTP; TH: MPTP + DHF + CQ: $0.48 \pm 0.06$ relative to CTR, $P<0.01$ vs. MPTP; Fig. $2 \mathrm{a}-\mathrm{c}, \mathrm{e}-\mathrm{g})$. As DHF binds to the TrkB extracellular domain and promotes its dimerization and autophosphorylation at tyrosine residues (Y705/706), we next tested the expression of total TrkB (t-TrkB), including full-length isoform (TrkB-FL) and a truncated protein lacking the tyrosine-kinase domain that is strikingly similar to the inactive TrkB-T1 isoform (TrkB-T1), and corresponding phosphorylated TrkB ( $p$-TrkB). The results showed that DHF treatment increased $\mathrm{p}$-TrkB level in both the striatum ( $n=5$ in each group; MPTP: $0.82 \pm 0.1$ relative to CTR, $P<0.05$ vs. CTR; MPTP + DHF: $1.21 \pm 0.1$ relative to CTR, $P<0.05$ vs. CTR, $P<$ 0.05 vs. MPTP; Fig. 2a, d) and midbrain (CTR: $n=5$ in each group; MPTP: $0.71 \pm 0.12$ relative CTR, $P<0.05$ vs. CTR; MPTP + DHF: $1.15 \pm 0.08$ relative to $C T R, P<0.05$ vs. $C T R, P<0.05$ vs. MPTP; Fig. $2 e, h)$, compared with those in MPTP-treated mice. Notably, chloroquine treatment displayed no effect on $\mathrm{p}$-TrkB expression (Fig. 2a, d, e, h).

\section{Chloroquine prevents the neuroprotective effects of DHF on dopaminergic neurons}

To further confirm whether autophagy is involved in protective effects of DHF on dopaminergic neurons after MPTP insult, immunohistochemistry assay was used to examine the $\mathrm{TH}$-positive neurons in the $\mathrm{SN}$ and terminals in the striatum. Results showed that the number of TH-positive neurons in the SN was significantly decreased after MPTP treatment. The administration of DHF markedly blocked the loss of $\mathrm{TH}$-positive neurons, while its protective effect was completely prevented by chloroquine treatment ( $n=4$ in each group; MPTP: $0.55 \pm 0.02$ relative to CTR, $P<0.05$ vs. CTR; MPTP + DHF: $0.89 \pm 0.05$ relative to CTR, $P>$ 0.05 vs. CTR, $P<0.05$ vs. MPTP; MPTP + DHF $+C Q: 0.51 \pm 0.03$ relative to CTR, $P<0.05$ vs. MPTP + DHF; Fig. 3a, c). Similarly, DHF treatment increased TH-positive terminals in the striatum of MPTPtreated mice, while chloroquine blocked these changes $(n=4$ in each group; MPTP: $0.66 \pm 0.02$ relative to CTR, $P<0.05$ vs. CTR; MPTP + DHF: $0.90 \pm 0.04$ relative to CTR, $P>0.05$ vs. CTR, $P<0.05$ vs. MPTP; MPTP + DHF + CQ: $0.65 \pm 0.04$ relative to $C T R, P<0.05$ vs. MPTP + DHF; Fig. 3a, b).

\section{DHF restores impaired autophagy in cell model of PD}

Aforementioned results indicate that DHF exerts beneficial effects on motor function and neuropathology via autophagy in MPTPinduced mouse model of PD. To further investigate the mechanism of autophagy in DHF-medicated therapeutic effects in $\mathrm{PD}$, we next introduced $\mathrm{N} 2 \mathrm{~A}$ cells treated with $\mathrm{MPP}^{+}$, a cell model of PD. The results from immunofluorescence experiments showed that LC3 puncta was significantly increased following DHF treatment in $\mathrm{MPP}^{+}$-treated cells, indicating that DHF increased the formation of autophagosomes (Fig. 4a). We further examined the expression of autophagy-related proteins, including beclin-1, P62 and LC3 that are commonly used to detect autophagy [31]. The results showed that the LC3II and beclin-1 were decreased, and the expression of P62 was increased in $\mathrm{MPP}^{+}$group, while DHF treatment restored the expression of P62, LC3II and beclin-1 to control levels, indicating that DHF rescued the $\mathrm{MPP}^{+}$-induced impairment of autophagy (P62: $n=6$ in each group; $\mathrm{MPP}^{+}: 1.47 \pm 0.08$ relative to CTR, $P<0.05$ vs. $\mathrm{CTR} \mathrm{MPP}^{+}+$ DHF: $1.02 \pm 0.09$ relative to CTR, $P>0.05$ vs. CTR, $P<0.05$ vs. $\mathrm{MPP}^{+}$; LC3II: $n=6$ in each group; $\mathrm{MPP}^{+}: 0.55 \pm 0.02$ relative to CTR, $P<0.05$ vs. CTR, MPP ${ }^{+}+$DHF: $0.93 \pm 0.02$ relative to CTR, $P>$ 0.05 vs. CTR, $P<0.05$ vs. $\mathrm{MPP}^{+}$; beclin-1: $n=4$ in each group; $\mathrm{MPP}^{+}: 0.77 \pm 0.02$ relative to $\mathrm{CTR}, P<0.05$ vs. $\mathrm{CTR}, \mathrm{MPP}^{+}+\mathrm{DHF}$ : $1.14 \pm 0.04$ relative to CTR, $P>0.05$ vs. CTR, $P<0.05$ vs. MPP ${ }^{+}$; Fig. $4 \mathrm{~b}-\mathrm{e})$. Autophagic flux is the entire process including autophagosome formation, delivery and fusion to lysosomes and subsequent degradation, which can be quantified by measuring the difference in the amount of LC3II in the presence and absence of saturating levels of inhibitors. To further determine the effects of DHF on autophagic flux, we tested the LC3II and P62 levels with or without the presence of chloroquine, an autophagy inhibitor that neutralizes lysosome PH [32]. We observed an accumulation of $\mathrm{P} 62$ after $\mathrm{MPP}^{+}$treatment, which suggests that autophagic flux is blocked, while DHF treatment repaired this interrupted autophagic flux as reflected by decreased P62 $(n=4$ in each group; $\mathrm{MPP}^{+}: 1.46 \pm 0.10$ relative to CTR, $P<0.05$ vs. $\mathrm{CTR} \mathrm{MPP}^{+}+$ DHF: $1.09 \pm 0.02$ relative to CTR, $P>0.05$ vs. CTR, $P<0.05$ vs. $\mathrm{MPP}^{+}$; Fig. 4b, c). As expected, P62 levels were increased in all the $\mathrm{CQ}$ treated groups compared with CTR and $\mathrm{MPP}^{+}+\mathrm{DHF}(n=4$ in each group; $\mathrm{MPP}^{+}+\mathrm{CQ}: 1.59 \pm 0.19$ relative to $\mathrm{CTR}, P<0.05$ vs. $\mathrm{CTR}, P<0.05$ vs. $\mathrm{MPP}^{+}+\mathrm{DHF} \mathrm{MPP}^{+}+\mathrm{DHF}+\mathrm{CQ}: 2.09 \pm 0.25$ relative to CTR, $P<0.05$ vs. CTR, $P<0.05$ vs. $\mathrm{MPP}^{+}+\mathrm{DHF} \mathrm{MPP}^{+}+$ $\mathrm{DHF}+\mathrm{CQ}+3-\mathrm{MA}: 1.57 \pm 0.22$ relative to CTR, $P<0.05$ vs. CTR, $P<$ 0.05 vs. $\mathrm{MPP}^{+}+\mathrm{DHF}$; Fig. 4f, g), which suggests CQ interrupted autophagy. We found that CQ administration increased LC3II levels in both the $\mathrm{MPP}^{+}$and $\mathrm{MPP}^{+}+\mathrm{DHF}$ groups, but the magnitude of the increase was significantly lower in the MPP group (the difference between $\mathrm{MPP}^{+}$and $\mathrm{MPP}^{+}+\mathrm{CQ}$ was 0.28) than in $\mathrm{MPP}^{+}+\mathrm{DHF}$ group (the difference between $\mathrm{MPP}^{+}+\mathrm{DHF}$ and $\mathrm{MPP}^{+}+\mathrm{DHF}+\mathrm{CQ}$ was 0.72$)(n=4$ in each group; Fig. $4 \mathrm{f}, \mathrm{h})$, which suggests that DHF increased autophagic flux. In addition, 3-MA, an autophagy inhibitor that blocks the early stage of autophagy by inhibiting class III PI3K, reduced the accumulation of LC3II in $\mathrm{MPP}^{+}+\mathrm{DHF}$ group caused by chloroquine $(n=4$ in each group; $\mathrm{MPP}^{+}+\mathrm{CQ}: 0.91 \pm 0.05$, relative to $\mathrm{CTR}, \mathrm{MPP}^{+}+\mathrm{DHF}$ $+\mathrm{CQ}: 1.7 \pm 0.16$ relative to $\mathrm{CTR}, P<0.05$ vs. $\mathrm{MPP}^{+}+\mathrm{CQ} \mathrm{MPP}^{+}+$

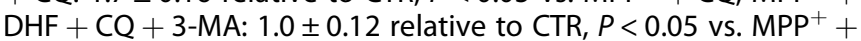
$\mathrm{DHF}+\mathrm{CQ}$; Fig. 4f, h), suggesting an increased autophagosome formation.

ANA-12 blocked the DHF-induced autophagy in PD cell model We have found that DHF treatment increases TrkB phosphorylation (Fig. 2) and autophagy (Fig. 4) in PD models. To determine whether the DHF-induced autophagy was dependent on TrkB activation, a specific TrkB antagonist ANA-12 was introduced. The results showed that ANA-12 completely blocked the DHF-induced increase of $\mathrm{p}$-TrkB in $\mathrm{MPP}^{+}$-treated N2A cells $(n=4$ in each group; $\mathrm{MPP}^{+}: 0.80 \pm 0.12 \mathrm{CTR}, P<0.05$ vs. $\mathrm{CTR} \mathrm{MPP}^{+}+\mathrm{DHF}: 1.56 \pm 0.24$ relative to CTR, $P<0.05$ vs. CTR, $P<0.05$ vs. $\mathrm{MPP}^{+} ; \mathrm{MPP}^{+}+\mathrm{DHF}+$ ANA-12: $0.70 \pm 0.13$ relative to $C T R, P<0.05$ vs. $C T R, P<0.05$ vs. $\mathrm{MPP}^{+}+$DHF; Fig. 5a, b). No significant difference was found in total TrkB expression (Fig. 5a, b). We also found that the DHFinduced autophagy was abrogated by ANA-12, as the expression 

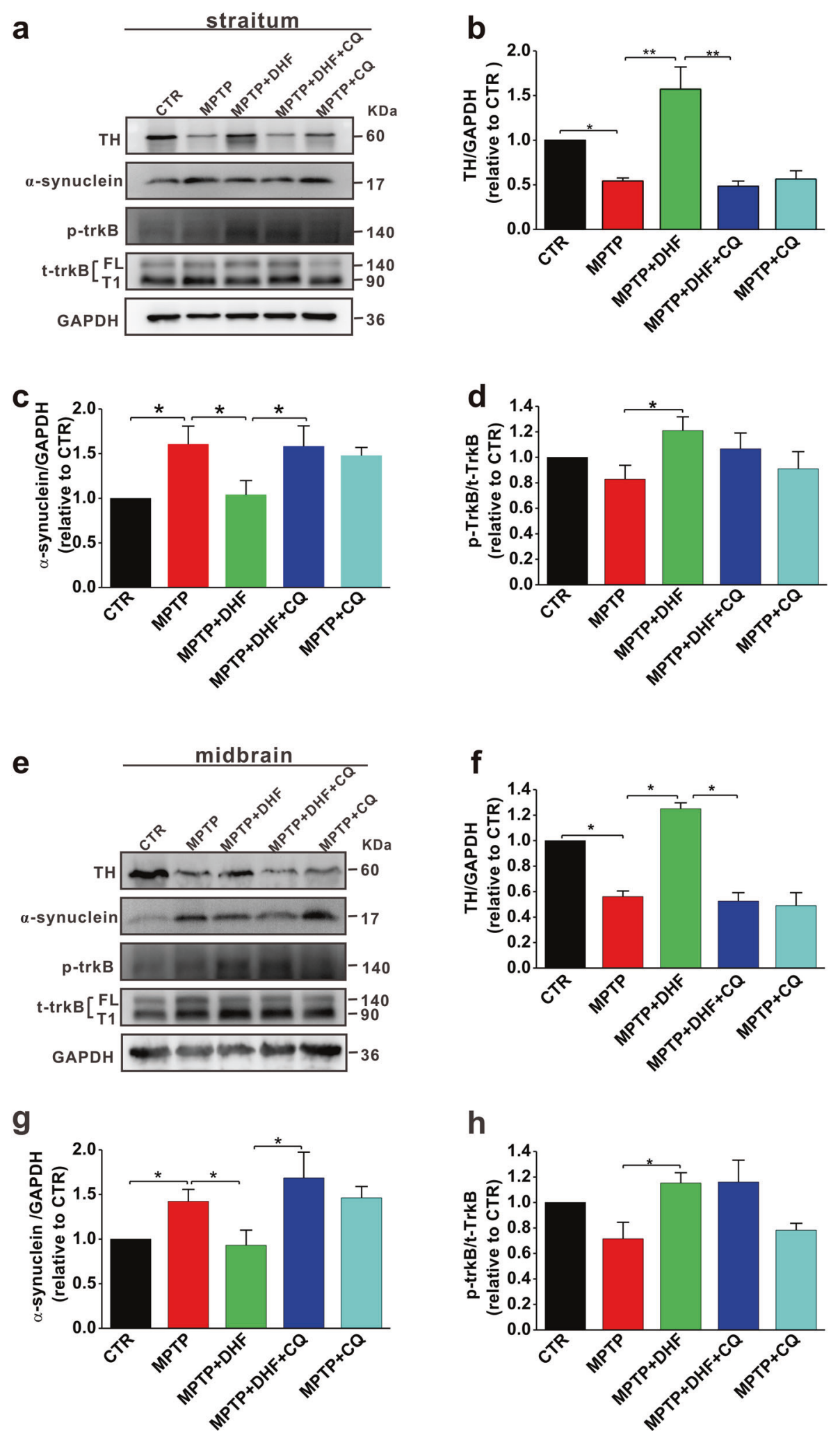

Fig. 2 Chloroquine (CQ) prevents the DHF-induced restoration of TH and decrease of a-synuclein accumulation in MPTP-induced mouse model of PD. a-d The protein levels of TH (b), $\alpha$-synuclein (c), and p-TrkB (d) assessed by western blot in the striatum from MPTP-induced mouse model of PD. e-h The protein levels of TH (f), $\alpha$-synuclein $(\mathbf{g})$ and $p$-TrkB (h) assessed by western blot in the midbrain from MPTPinduced mouse model of PD. Data are expressed as mean \pm SEM. ${ }^{*} P<0.05,{ }^{* *} P<0.01$. 
a
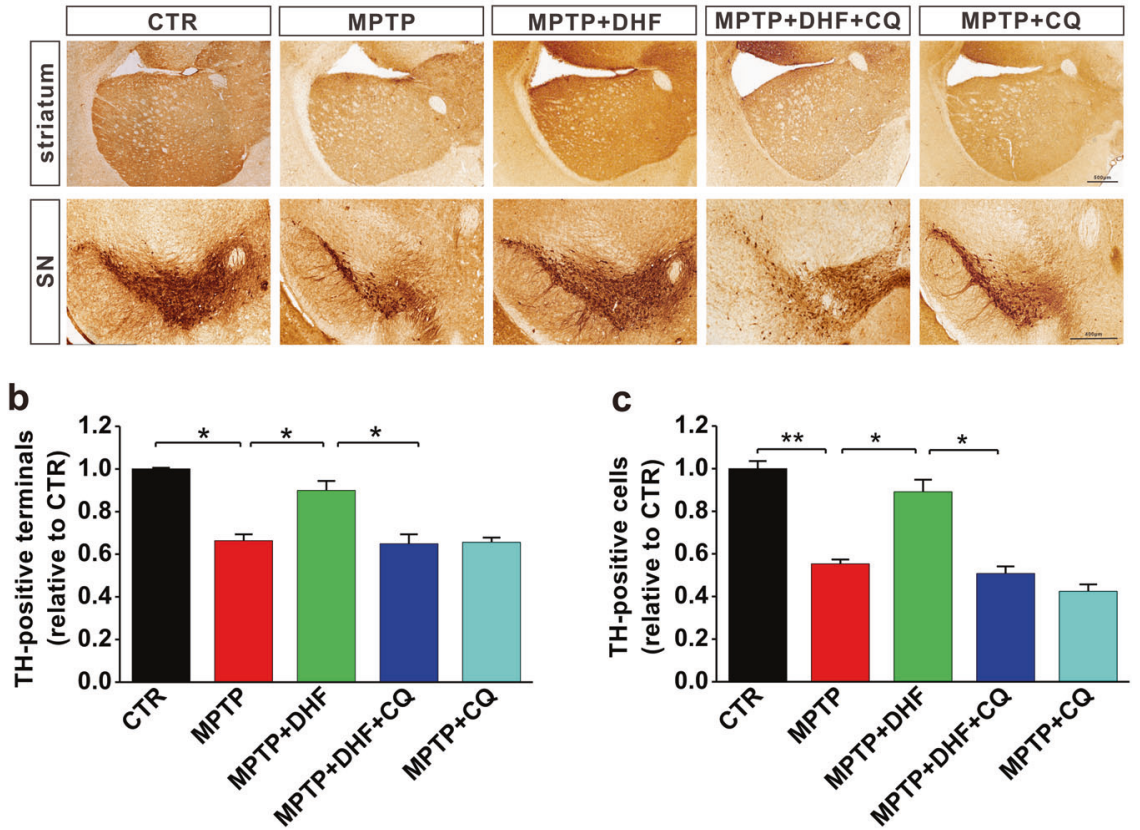

Fig. 3 Chloroquine (CQ) prevents the protective effects of DHF on the loss of dopaminergic neurons in MPTP-induced mouse model of PD. a Representative photomicrographs of TH-positive terminals in striatum and TH-positive neurons in midbrain. $\mathbf{b}$, $\mathbf{c}$ Quantitative analysis of TH-positive terminals in the striatum (b) and TH-positive cells in the midbrain (c). Data are expressed as mean \pm SEM. ${ }^{*} P<0.05,{ }^{* *} P<0.01$.

of both P62 and LC3II were increased, indicating autophagosomes were accumulated and autophagy pathway was interrupted in late stage (P62: $n=4$ in each group; $\mathrm{MPP}^{+}: 1.48 \pm 0.08$ relative to CTR, $P<0.05$ vs. CTR; $\mathrm{MPP}^{+}+\mathrm{DHF}: n=4,1.06 \pm 0.07$ relative to $\mathrm{CTR}$, $P>0.05$ vs. CTR, $P<0.05$ vs. $\mathrm{MPP}^{+} ; \mathrm{MPP}^{+}+\mathrm{DHF}+\mathrm{ANA}-12: 1.67 \pm$ 0.02 relative to CTR, $P<0.05$ vs. $\mathrm{MPP}^{+}+\mathrm{DHF}$; LC3II: $n=4$ in each group; $\mathrm{MPP}^{+}: 0.80 \pm 0.04$ relative to CTR, $P<0.05$ vs. CTR; $\mathrm{MPP}^{+}+$ DHF: $1.20 \pm 0.09$ relative to CTR, $P>0.05$ vs. CTR; $P<0.05$ vs. $\mathrm{MPP}^{+}$; $\mathrm{MPP}^{+}+\mathrm{DHF}+\mathrm{ANA}-12: 1.67 \pm 0.14 \mathrm{CTR}, \mathrm{P}<0.05 \mathrm{vs} . \mathrm{MPP}^{+}+\mathrm{DHF}^{\prime}$ Fig. $5 \mathrm{a}, \mathrm{c}, \mathrm{d}$ ). These results suggest that the DHF-induced autophagy may be executed through binding to TrkB receptors.

DHF promotes autophagy through ERK-LKB1-AMPK pathway Previous studies have shown that DHF binds to TrkB receptor and activates PI3K/Akt, MAPK, and PLC- $\gamma$ signaling cascades [17]. Therefore, we next wanted to examine the downstream signal pathways that may be involved in DHF-mediated autophagy. Consistent with previous report [33], we found that the expression of phosphorylated ERK1/2 (p-ERK1/2) was significantly increased in $\mathrm{MPP}^{+}$-treated cells compared with control, while DHF treatment restored the p-ERK $1 / 2$ expression to control level $(n=5$ in each group; $\mathrm{MPP}^{+}: 34.7 \pm 2.25$ relative to $\mathrm{CTR}, P<0.001$ vs. CTR; $\mathrm{MPP}^{+}$ + DHF: $6.17 \pm 0.02$ relative to CTR, $P<0.001$ vs. MPP $^{+}$; Fig. $\left.6 a, b\right)$. To further determine whether ERK1/2 played a critical role in autophagy, we introduced ERK1/2 inhibitor U0126 and measured the expression of P62 and LC3II. The results showed that U0126 treatment following $\mathrm{MPP}^{+}$markedly reduced $\mathrm{p}$-ERK $1 / 2$ expression $\left(n=5\right.$ in each group; $\mathrm{MPP}^{+}: 34.7 \pm 2.25$ relative to $\mathrm{CTR}, P<0.001$ vs. $\mathrm{CTR} \mathrm{MPP}^{+}+\mathrm{U} 0126: 23.0 \pm 1.1$ relative to $\mathrm{CTR}, P<0.05$ vs. $\mathrm{MPP}^{+}$; Fig. 6a, b), concomitantly with increased LC3\| and decreased P62 (LC3II: $n=5$ in each group; $\mathrm{MPP}^{+}$: $0.67 \pm 0.08$ relative to $C T R, P<0.001$ vs. $C T R$; $\mathrm{MPP}^{+}+\mathrm{U} 0126: 0.98 \pm 0.04$ relative to $C T R, P<0.05$ vs. $\mathrm{MPP}^{+} ; \mathrm{MPP}^{+}+\mathrm{DHF}: 1.09 \pm 0.04$ relative to $C T R, P<0.001$ vs. $\mathrm{MPP}^{+} ; \mathrm{P} 62: n=5$ in each group; $\mathrm{MPP}^{+}: 2.51 \pm$ 0.17 relative to $C T R, P<0.001$ vs. $\mathrm{CTR} \mathrm{MPP}^{+}+\mathrm{U} 0126: 1.35 \pm 0.10$ relative to $\mathrm{CTR}, P<0.05$ vs. $\mathrm{MPP}^{+} ; \mathrm{MPP}^{+}+\mathrm{DHF}: 1.08 \pm 0.06$ relative to $C T R, P<0.001$ vs. MPP ${ }^{+}$; Fig. 6 a, e, f), indicating that suppressing ERK1/2 activation induced autophagy.

Since AMPK and mTOR pathway play an important role in the regulation of autophagy, we then investigated whether AMPK and mTORC1 were involved in DHF-induced autophagy. The results showed that the phosphorylated AMPK ( $p-A M P K)$ was significantly decreased in $\mathrm{MPP}^{+}$-treated cells $(n=5$ in each group; $\mathrm{MPP}^{+}: 0.49 \pm 0.09$ relative to $\mathrm{CTR}, P<0.05$ vs. CTR; Fig. $6 \mathrm{a}$, d), while $\mathrm{p}-\mathrm{mTOR}$ remained unchanged ( $n=4$ in each group; Fig. S1a, c). DHF treatment increased p-AMPK to control level $(n=5$ in each group; $\mathrm{MPP}^{+}: 0.49 \pm 0.09$ relative to $\mathrm{CTR}, \mathrm{MPP}^{+}+\mathrm{DHF}$ : $0.98 \pm 0.05$ relative to $\mathrm{CTR}, P<0.05$ vs. $\mathrm{MPP}^{+}$; Fig. $6 \mathrm{a}$, d). As the suppression of ERK1/2 activation induced autophagy, we next wanted to determine whether there is a link between $\mathrm{p}$-ERK1/2 and p-AMPK. The results showed that p-AMPK (Thr172) was increased after treatment with ERK1/2 inhibitor U0126, indicating ERK1/2 may negatively regulated AMPK phosphorylation $\left(\mathrm{MPP}^{+}\right.$: $0.49 \pm 0.09$ relative to $\mathrm{CTR}, \mathrm{MPP}^{+}+\mathrm{U} 0126: 1.0 \pm 0.02$ relative to CTR, $P<0.05$ vs. MPP ${ }^{+}$; Fig. $\left.6 \mathrm{a}, \mathrm{d}\right)$. The phosphorylation of AMPK can be regulated by constitutively active kinase STK11/LKB1 [34]. The previous study has shown that ERK1/2 can inhibit LKB1 through phosphorylation of Ser428 residue, and LKB1, in turn, acts upstream of the AMPK/ULK1/ATG1 signal axis to regulate autophagy $[34,35]$. We, therefore, tested the expression of p-LKB1 (Ser428). In agreement with previous study, DHF or U0126 attenuated the level of $\mathrm{p}$-ERK $1 / 2$ and $\mathrm{p}$-LKB1 $(n=5$ in each group; $\mathrm{MPP}^{+}: 1.54 \pm 0.03$ relative to CTR, $P<0.05$ vs. CTR; $\mathrm{MPP}^{+}+\mathrm{U} 0126$ : $1.13 \pm 0.08$ relative to $\mathrm{CTR}, P<0.05$ vs. $\mathrm{MPP}^{+} ; \mathrm{MPP}^{+}+\mathrm{DHF}: 1.10 \pm$ 0.065 relative to $\mathrm{CTR}, P<0.05$ vs. $\mathrm{MPP}^{+}$; Fig. $\left.6 \mathrm{a}, \mathrm{C}, \mathrm{d}\right)$. Alternatively, AMPK (Thr172) is also phosphorylated by the $\mathrm{Ca}^{2+}$-activated protein kinase CaMKK2/CaMKK $\beta$ [36], which can be activated by TrkB downstream PLC- $\gamma-\mathrm{IP3}_{-}-\mathrm{Ca}^{2+}$ signaling. However, we found that PLC- $\gamma$ inhibitor U73122 had no effect on DHF-induced autophagy, suggesting that PLC $-\gamma$ may not involve in this process (Fig. S2). Together, these findings suggest that DHF decreased ERK1/2 phosphorylation, and subsequently regulated LKB1 and AMPK activation, thereby promotes autophagy in PD models. 
a

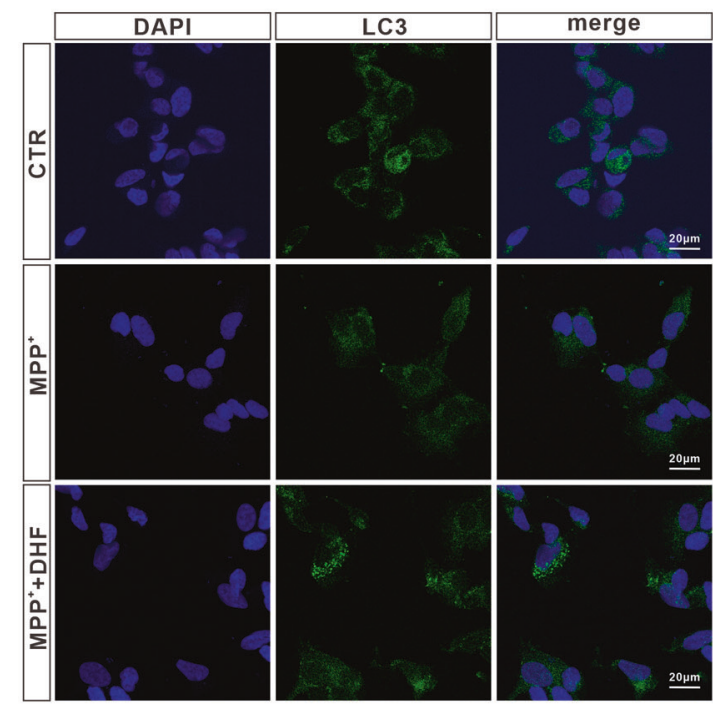

b

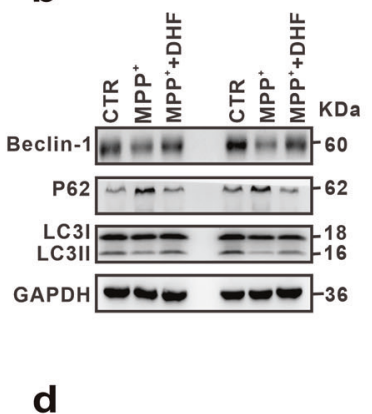

C

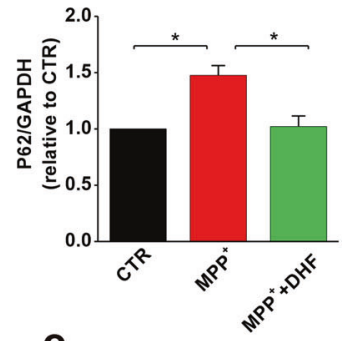

e
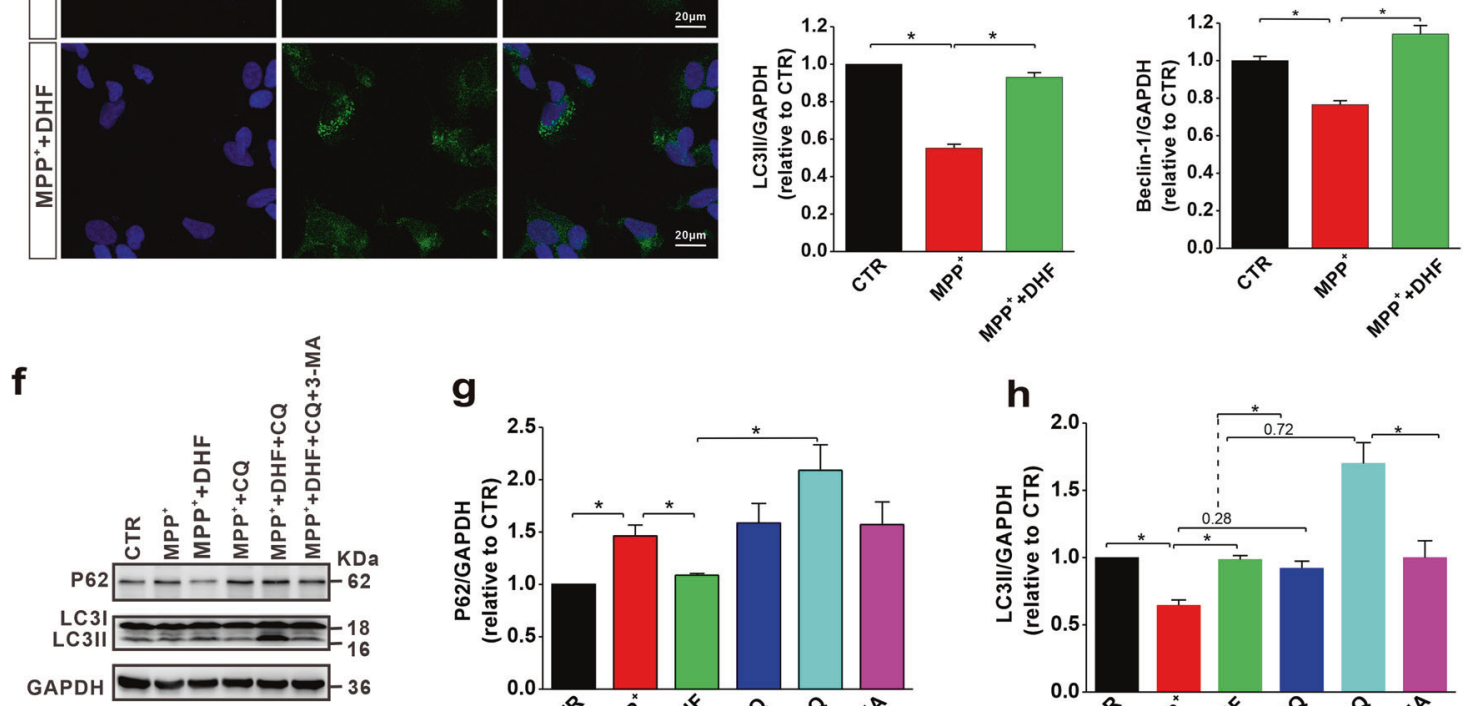

g

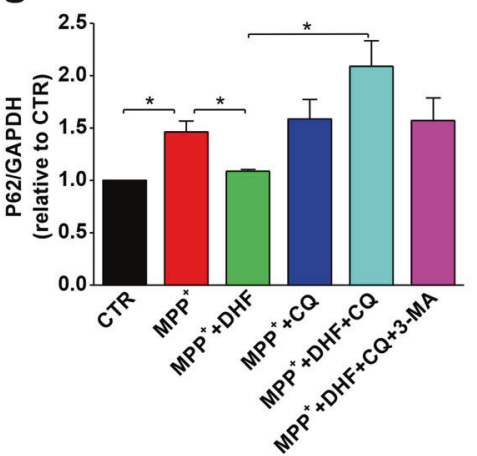

h

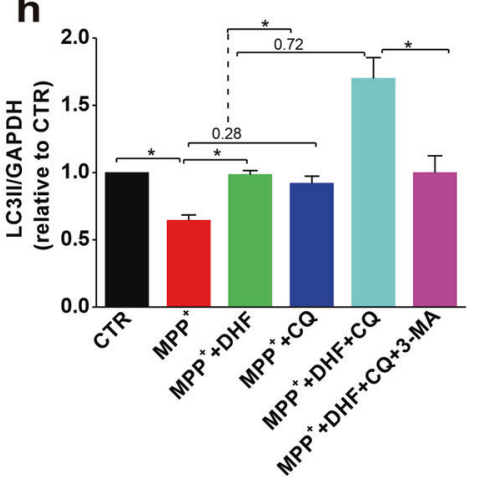

Fig. 4 DHF induces autophagy in MPP ${ }^{+}$-treated N2A cells. a Immunofluorescence analysis of LC3 to evaluate autophagy in N2A cells. b-e The protein levels of P62 (c), LC3 (d), and Beclin-1 (e) assessed by western blot in N2A cells subjected to MPP ${ }^{+}$treatment with or without DHF. $\mathbf{f}-\mathbf{h}$ The protein levels of P62 (g) and LC3 (h) assessed by western blot in MPP ${ }^{+}$-treated N2A cells with or without co-administration of DHF and CQ or 3-MA. Data are expressed as mean \pm SEM. ${ }^{*} P<0.05$.

\section{DISCUSSION}

In the present study, we first confirm that DHF has protective effects on motor function and neuropathology in MPTP-induced PD model mice, and also demonstrate that DHF exerts protective effects by restoring impaired autophagy through ERK-LKB1-AMPK pathway (Fig. 7).

As a mimetic of BDNF, DHF has been gained a lot of attention as a therapeutic target in various BDNF-implicated human diseases, especially in neurological disorders. In previous studies, the protective effects of DHF were mainly focus on its anti-apoptosis, anti-inflammatory, and anti-oxidative function $[37,38]$. It has been well documented that autophagy is a biological process that requires a sequential stimulation of molecular events including autophagosome formation, delivery to the lysosome and eventual fusion with lysosomes to degradation [39]. The impairment of autophagy is thought to contribute to the pathogenesis of many neurodegenerative diseases including PD [4-6]. Consistently, we here have reported that the autophagy is impaired in PD models, and importantly, we have found that DHF induces autophagy and enhances a-synuclein degradation through autolysosome pathway (Fig. 4). This finding is supported by a recent study that DHF improves autophagy and alleviates the depressive behaviors in animal models [40]. The Atg1/ULK1 is a central component and plays a crucial role in the process of autophagy especially in the autophagosome formation during which ULK1, ATG13, ATG101, and FIP200 assemble into ULK1 complex and regulate autophagosome formation [41]. ULK1 is mainly regulated by mTOR and AMPK [42]. In the present study, we have found that DHF increases AMPK phosphorylation at Thr172, and then increases phosphorylation of ULK1 at Ser555 (Fig. S1a, b), thereby increases autophagy in PD.

DHF promotes TrkB receptor dimerization and autophosphorylation, which causes the activation of PI3K/Akt, MAPK, and PLC- $\gamma$ signaling cascades [12]. Here we have found that DHF triggers autophagy induction, which is mediated through the TrkB phosphorylation and subsequent regulation of MAPK pathway. Previous studies have shown that the LKB1-AMPK signaling axis, which could be regulated by ERK1/2 [43], is a key regulator of autophagy and plays an important role in cellular metabolic sensing and regulation of cell growth, proliferation, and survival $[34,35]$. We here report that DHF reduces ERK1/2 phosphorylation in $\mathrm{MPP}^{+}$-treated N2A cells, and consequently activates LKB1 and AMPK (Fig. 6). At present study, DHF inhibited the activation of ERK1/2, which is consistent with other studies that DHF also reduced the ERK signaling $[22,44]$. Nonetheless, some contradictory results show that the ERK1/2 is activated after DHF 
a

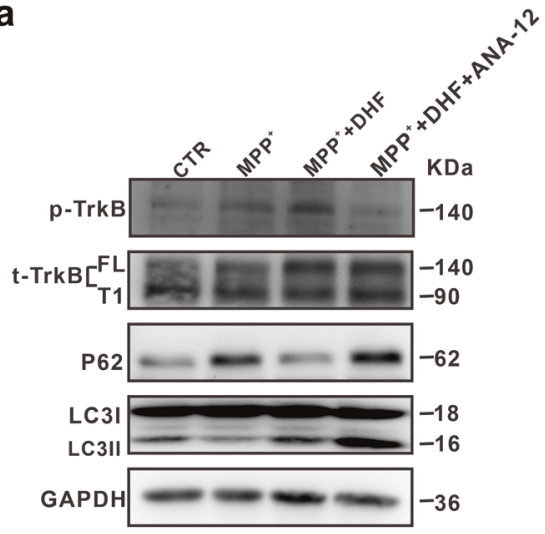

C

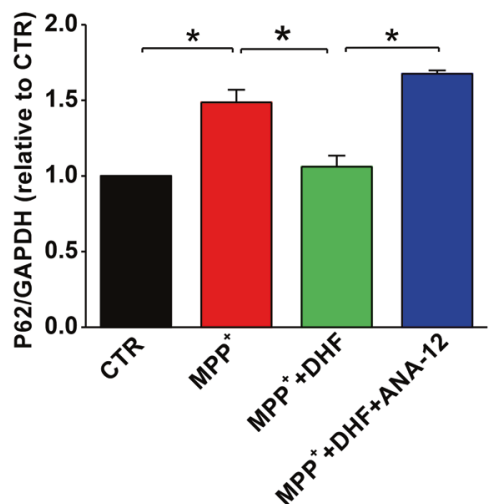

b

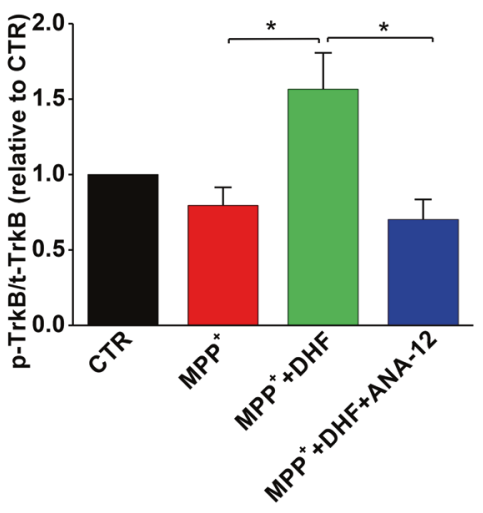

d

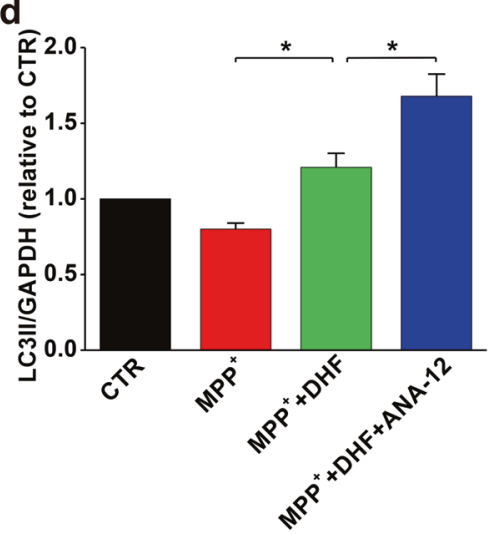

Fig. 5 TrkB inhibitor ANA-12 blocks the DHF-induced autophagy. a Sequential immunoblotting of cell lysates. b-d The protein levels of p-TrkB (b), P62 (c) and LC3 (d) assessed by western blot in MPP ${ }^{+}$-treated N2A cells with or without co-administration of DHF and ANA-12. Data are expressed as mean \pm SEM. ${ }^{*} P<0.05$.

treatment $[17,45]$. One possibility is that DHF reduces ROS with its anti-oxidative property [23], which subsequently inhibited ERK1/2 activation [46].

As a mimetic of BDNF, DHF exerts similar effects as BDNF. The role of $\mathrm{BDNF}$ in the regulation of autophagy has been extensively studied [47]. Generally, BDNF was thought to suppress autophagy. It has been reported that the regulation of autophagy in the brain is paralleled by changes in BDNF levels, and BDNF regulates synaptic plasticity by suppressing the autophagic flux in neurons [48]. However, some studies have shown that BDNF-TrkB promotes autophagy flux [24, 25]. These discrepancies may be accounted for by different cell types and pathological conditions. In the present study, we have found DHF promotes autophagic flux in PD models. DHF specifically binds to TrkB receptor, while BDNF acts on both the p75 neurotrophic receptor and the TrkB receptor. Moreover, the tyrosine phosphorylated residues on TrkB intracellular domain and their relative abundance are different after BDNF and DHF stimulations, which may exert different downstream effects [18]. Given the different characteristics of DHF and BDNF, determining the different roles and potential mechanisms of DHF and BDNF in the regulation of autophagy is essential in future research.

In conclusion, this study shows that DHF alleviates motor deficits and reduces the loss of dopaminergic neurons in MPTPinduced mouse model of PD. These protective effects are involved in the induction of autophagy by increased phosphorylation of AMPK via ERK1/2- regulated LKB1 activation. These findings indicate that potential therapeutic capabilities of DHF to slow the progression of PD by improving impaired autophagy.

\section{MATERIALS AND METHODS}

Animals

C57BL/6 male mice $(25-30 \mathrm{~g})$ were purchased from Charles River Laboratories and raised at the animal care center of Children's Hospital affiliated to Chongqing Medical University. Animals were housed at room temperature in plastic cages with free access to food and water under a $12 \mathrm{~h} / 12 \mathrm{~h}$ light/dark cycle. The mice were randomly divided into 5 groups for different treatment. All animal experiments were conducted in accordance with the Chongqing Science and Technology Commission guidelines and approved by the Chongqing Medical University Animal Care Committee, and every effort was made to minimize both animal suffering and number of animals used.

\section{Drugs and treatments}

MPTP, MPP ${ }^{+}, 3-M A$, ANA-12, and U73122 were purchased from SigmaAldrich. Chloroquine was purchased from MCE. U0126 was purchased from Abcam. DHF was purchased from Tokyo Chemical Industry. MPTP and chloroquine were dissolved in sterile saline. DHF was dissolved in sterile saline containing $10 \%$ ethanol. MPTP groups were administrated with MPTP (30 mg/kg, i.p.) once a day for 5 days. DHF $(5 \mathrm{mg} / \mathrm{kg}$, i.p.) and chloroquine $(50 \mathrm{mg} / \mathrm{kg}$, i.p.) were injected once a day during the whole process of experiments. The control groups received the same volume of sterile saline with or without $10 \%$ ethanol.

\section{Antibodies}

Following primary antibodies were used in the present study: anti-GAPDH (1:5000; arigo, ARG10112), anti-TH (1:1000; BD biosciences, 612300), anti- $\alpha$ synuclein (1:1000; BD bioscicences, 610786), anti-p-TrkB (1:1000; abcam, ab229908), anti-trkB (1:1000; Cell Signaling Technology, 4603 T), anti-LC3A/B (1:1000; Cell Signaling Technology, 12741S), anti-P62 (1:1000; proteintech, 18420-1-AP), anti-beclin-1 (1:1000; Cell Signaling Technology, 3738S), anti-p- 

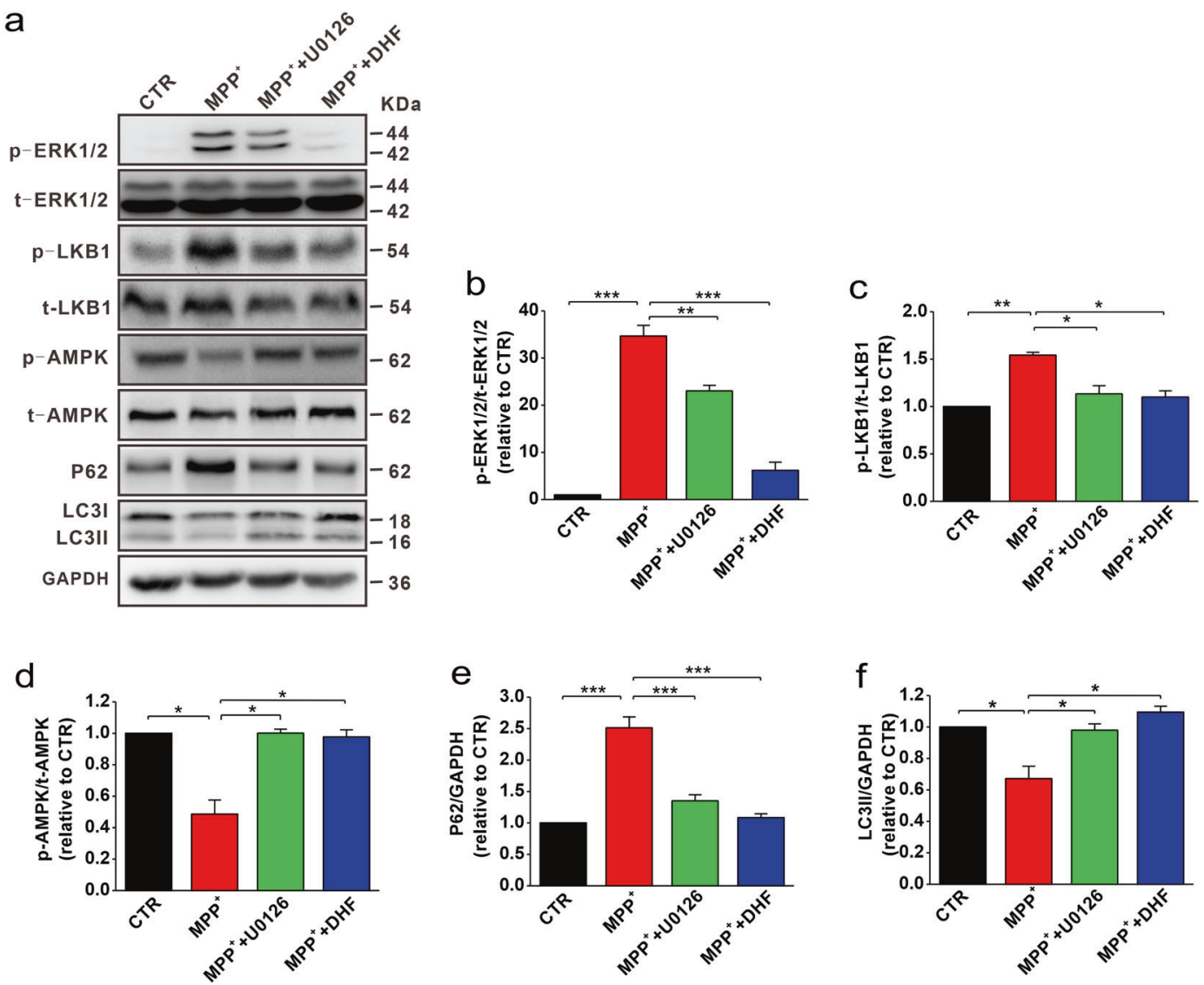

Fig. 6 DHF-promoted autophagy is dependent on ERK-LKB1-AMPK pathway. a Sequential immunoblotting of cell lysates. $\mathbf{b}-\mathbf{f}$ The protein levels of p-ERK1/2 (b), p-LKB1 (c), p-AMPK (d), P62 (e) and LC3 (f) assessed by western blot in MPP ${ }^{+}$-treated N2A cells with or without coadministration of DHF and U0126. Data are expressed as mean \pm SEM. ${ }^{*} P<0.05,{ }^{* *} P<0.01,{ }^{* * *} P<0.001$.

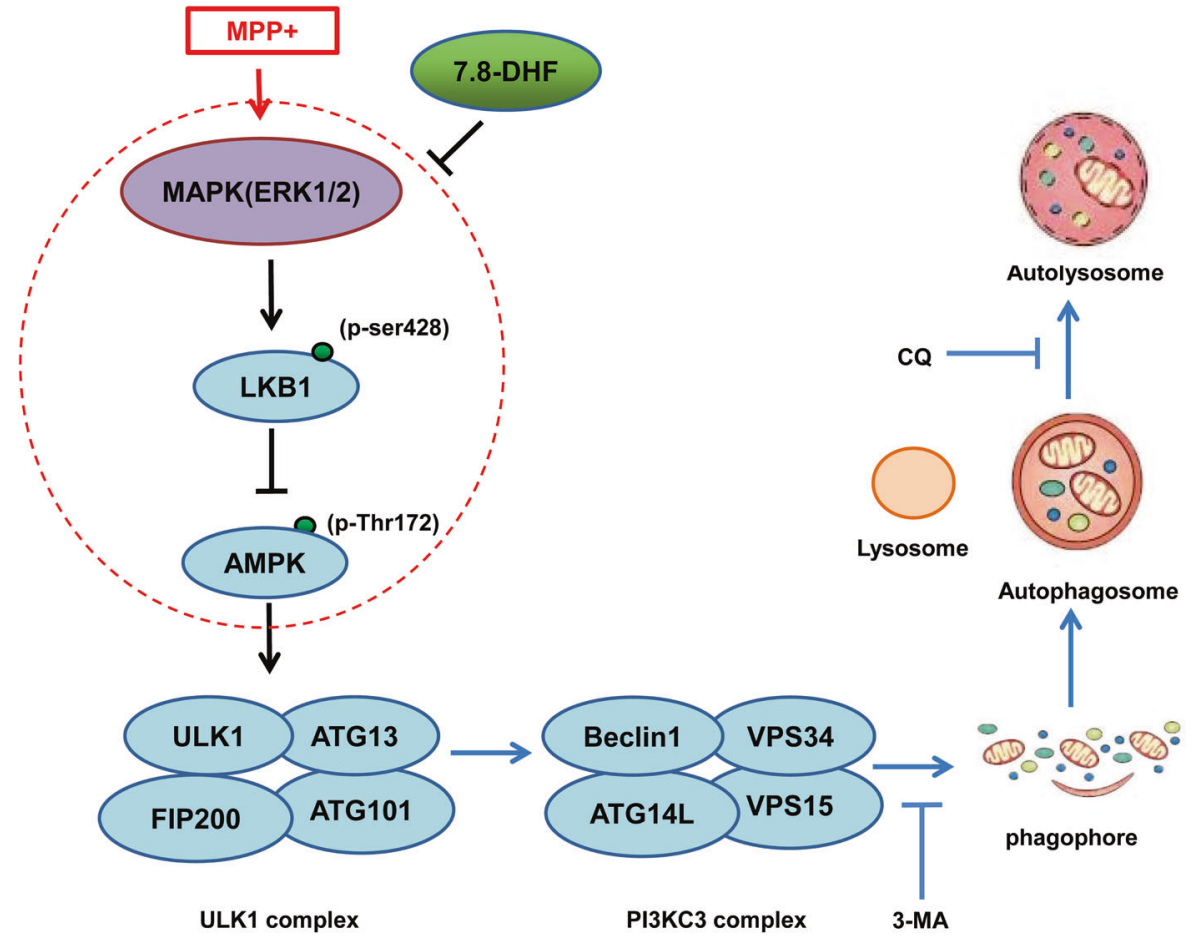

Fig. 7 Proposed model for DHF-improved autophagy in PD. ERK1/2 was highly phosphorylated (p-ERK1/2) in MPP ${ }^{+}$-treated PD model cells, which led to LKB1 phosphorylation at Ser428 and impaired the ability of LKB1 to phosphorylate AMPK at Thr172, thereby blocking the activation of AMPK and AMPK-initiated autophagy. DHF inhibited the ERK1/2 activation and restored the ability of LKB1 in activating AMPK and autophagy. 
ERK1/2 (1:1000; Cell Signaling Technology, 4370S), anti-ERK1/2 (1:1000; Cell Signaling Technology, 4695), anti-p-LKB1 (1:1000; Cell Signaling Technology, 3482S), anti-LKB1 (1:1000; Cell Signaling Technology, 3047), anti-p-AMPK (1:1000; Cell Signaling Technology, 2535S), anti-p-mTOR (1:1000; Cell Signaling Technology, 5536T), anti-AMPK (1:1000; Cell Signaling Technology, 2532) and anti-p-ULK1 (1:1000; Cell Signaling Technology, 5869).

\section{Rotarod test}

The rotarod test was performed to analyze motor function in PD model mice. Briefly, mice were received 4 rounds of training on the rotarod after 2-week drug administration. In the first two rounds of training, mice were trained at constant speed of $20 \mathrm{rpm}$ for $3 \mathrm{~min}$. In the following two rounds, mice were trained at constant speed of $20 \mathrm{rpm}$ for $3 \mathrm{~min}$ in rocking mode, in which the rotarod changed rotation direction from clockwise to counterclockwise every 3 turns. On the second day, mice were given formal rotarod test with rotation speed at $20 \mathrm{rpm}$ in rocking mode. The test consisted of 10 times with time interval of $20 \mathrm{~min}$, and the time that the mouse remained on the rod during each test was recorded. Maximum test time (cut-off limit) was $300 \mathrm{~s}$.

\section{Pole test}

The pole test was used to measure the degree of bradykinesia, as previously described with some modifications [49]. In brief, mice were put upward on the top of a rough surfaced pole (length: $50 \mathrm{~cm}$, diameter: $1.5 \mathrm{~cm}$ ), and the latency to descend the pole was measured. Trials were excluded if the mouse fell or slid down the pole rather than climbed down. Each mouse was trained for 2 trials on the first day and tested for 5 trials on the second day. The lowest latency to descend the pole was analyzed.

\section{Wire suspension test}

Wire suspension test was used to test motor function in PD model mice. The whole set contains a wire and two platforms. The wire was fixed horizontally to two platforms and was kept $80 \mathrm{~cm}$ long, $25 \mathrm{~cm}$ high. Each mouse was suspended with its paws on the middle of the wire, and the time taken to reach the platform is recorded. Mice were trained with two trials on the first day and tested for five trials on the second day. The shortest latency to reach the platform was analyzed. Trials were excluded if the mouse fell from wire or stayed still. Maximum test time (cut-off limit) was $120 \mathrm{~s}$.

\section{Western blotting}

Brain tissues or N2A cells that were obtained from Professor Weihong Song (University of British Columbia, Vancouver, Canada), were lysed on ice with lysis buffer (Keygen Biotech, KGP702), and then the extracts were centrifuged at $12,000 \times g$ for $15 \mathrm{~min}$ at $4{ }^{\circ} \mathrm{C}$. The supernatant was collected, and protein concentration was measured by BCA protein assay kit (Thermo Fisher Scientific, Waltham, MA, USA). Equal amount of protein samples was mixed with $5 \times$ sample buffer, boiled at $95^{\circ} \mathrm{C}$ for $5 \mathrm{~min}$, and separated on $10-15 \%$ sodium dodecyl sulfate-polyacrylamide gel electrophoresis (SDSPAGE). Proteins were then transferred to polyvinylidene fluoride (PVDF) membranes (Bio-Rad, Hercules, CA, USA). Membranes were blocked with $5 \%$ nonfat milk in Tris-buffered saline containing $0.1 \%$ Tween-20 (TBST) for $1 \mathrm{~h}$ at room temperature, and then incubated overnight at $4{ }^{\circ} \mathrm{C}$ with primary antibody. After washing 3 times in TBST, membranes were incubated with secondary antibody for $1 \mathrm{~h}$ at room temperature. After another 3 washes with TBST, protein band was visualized in the Bio-Rad Imager with ECL Western blotting substrate (Pierce). Immunoblotting with anti-GAPDH was used to control equal loading and protein quality. The band intensity was quantified by the Bio-Rad Quantity One software.

\section{TH Staining and quantification}

Mice were anesthetized with urethane $(1.5 \mathrm{~g} / \mathrm{kg}$, i.p.), and then perfused with phosphate-buffered saline (PBS) followed by $4 \%$ paraformaldehyde. The brain was removed and fixed in $4 \%$ paraformaldehyde and dehydrated in $30 \%$ sucrose solution, then sectioned into $30 \mu \mathrm{m}$ slices with Leica cryostat. Sections were rinsed in PBS and then incubated in $3 \%$ $\mathrm{H}_{2} \mathrm{O}_{2}$ for 10 min to eliminate the activity of endogenous peroxidase. After washing 3 times in PBS, brain sections were incubated in PBS solution containing $5 \%$ BSA and $0.2 \%$ Triton $\mathrm{X}-100$ for $1 \mathrm{~h}$ at room temperature, then incubated overnight in anti-TH (1:500 dilution) at $4{ }^{\circ} \mathrm{C}$. Finally, a biotinylated goat anti-mouse secondary antibody (Zhongshan Golden Bridge Biotechnology, PV9000) and diaminobenzidine (Zhongshan Golden
Bridge Biotechnology, ZLI-9018) were used according to the manufacturer's instruction to detect the immunoreactivity. The quantification of THpositive cells in the $\mathrm{SN}$ and TH-positive neuronal terminals in the striatum were counted at six-section intervals throughout the entire extent of SN and striatum, as previously described [23].

\section{Immunofluorescence and confocal microscopy}

$\mathrm{N} 2 \mathrm{~A}$ cells were treated with $\mathrm{MPP}^{+}$and DHF for $24 \mathrm{~h}$, fixed in $4 \%$ paraformaldehyde for $20 \mathrm{~min}$. After washing 3 times with PBS, cells were permeabilized with $0.3 \%$ Triton X-100 in PBS for 30 min and blocked with $5 \%$ BSA for $1 \mathrm{~h}$ at room temperature. Then, cells were incubated with rabbit anti-LC3 antibody overnight at $4{ }^{\circ} \mathrm{C}$ followed by Alexa Fluor 488 (Invitrogen, A11034) conjugated secondary antibody for $1 \mathrm{~h}$ at room temperature. Finally, N2A cells were counterstained with DAPI (SigmaAldrich, D9542) and imaged with a confocal microscope.

\section{Statistical analysis}

All data were expressed as mean \pm SEM. The differences of rotarod test were analyzed by two-way ANOVA, with treatment (group) as the between-subjects factor and test as the within-subjects factor. The data of all other experiments were analyzed by one-way ANOVA, followed by Tukey's post hoc test. Significance level was set at $P<0.05$.

\section{DATA AVAILABILITY}

The datasets used and analyzed during the current study are available from the corresponding author on reasonable request.

\section{REFERENCES}

1. Kalia LV, Lang AE. Parkinson's disease. Lancet. 2015;386:896-912.

2. Ascherio A, Schwarzschild MA. The epidemiology of Parkinson's disease: risk factors and prevention. Lancet Neurol. 2016;15:1257-72.

3. Michel $P$, Hirsch $E$, Hunot $S$. Understanding dopaminergic cell death pathways in Parkinson disease. Neuron. 2016;90:675-91.

4. Hara T, Nakamura $K$, Matsui $M$, Yamamoto A, Nakahara $Y$, Suzuki-Migishima $R$ et al. Suppression of basal autophagy in neural cells causes neurodegenerative disease in mice. Nature. 2006;441:885-9.

5. Komatsu M, Waguri S, Chiba T, Murata S, Iwata J, Tanida I, et al. Loss of autophagy in the central nervous system causes neurodegeneration in mice. Nature. 2006;441:880-4.

6. Sato S, Uchihara T, Fukuda T, Noda S, Kondo H, Saiki S, et al. Loss of autophagy in dopaminergic neurons causes Lewy pathology and motor dysfunction in aged mice. Sci Rep. 2018;8:2813.

7. Lachenmayer M, Yue ZJA. Genetic animal models for evaluating the role of autophagy in etiopathogenesis of Parkinson disease. Autophagy. 2012;8:1837-8.

8. Auluck $P$, Caraveo G, Lindquist S.a-Synuclein: membrane interactions and toxicity in Parkinson's disease. Annu Rev. Cell Dev. Biol. 2010;26:211-33.

9. Webb J, Ravikumar B, Atkins J, Skepper J, Rubinsztein DC. Alpha-Synuclein is degraded by both autophagy and the proteasome. J. Biol. Chem. 2003:278:25009-13.

10. Ebrahimi-Fakhari D, McLean P, Unni VK. Alpha-synuclein's degradation in vivo: opening a new (cranial) window on the roles of degradation pathways in Parkinson disease. Autophagy. 2012;8:281-3.

11. Kim D, Lim H, Kawasaki I, Shim Y, Vaikath N, El-Agnaf $O$, et al. Anti-aging treatments slow propagation of synucleinopathy by restoring lysosomal function. Autophagy. 2016;12:1849-63.

12. Jang SW, Liu X, Yepes $M$, Shepherd KR, Miller GW, Liu $Y$, et al. A selective TrkB agonist with potent neurotrophic activities by 7,8-dihydroxyflavone. Proc Natl Acad Sci USA. 2010;107:2687-92.

13. Balaratnasingam $S$, Janca $A$. Brain derived neurotrophic factor: a novel neurotrophin involved in psychiatric and neurological disorders. Pharmacol Ther. 2012;134:116-24.

14. Nagahara AH, Tuszynski MH. Potential therapeutic uses of BDNF in neurological and psychiatric disorders. Nat Rev Drug Disco. 2011;10:209-19.

15. Ochs G, Penn R, York M, Giess R, Beck M, Tonn J, et al. A phase I/II trial of recombinant methionyl human brain derived neurotrophic factor administered by intrathecal infusion to patients with amyotrophic lateral sclerosis. Amyotroph. Lateral Scler. Other Mot. Neuron Disord. 2000;1:201-6.

16. Thoenen $\mathrm{H}$, Sendtner M. Neurotrophins: from enthusiastic expectations through sobering experiences to rational therapeutic approaches. Nat. Neurosci. 2002:5:1046-50. 
17. Emili M, Guidi S, Uguagliati B, Giacomini A, Bartesaghi R, Stagni F. Treatment with the flavonoid 7,8-dihydroxyflavone: a promising strategy for a constellation of body and brain disorders. Crit Rev Food Sci Nutr. 2020;11:1-38 (2020).

18. Liu C, Chan CB, Ye K. 7,8-dihydroxyflavone, a small molecular TrkB agonist, is useful for treating various BDNF-implicated human disorders. Transl. Neurodegeneration. 2016;5:2

19. Liu X, Obianyo O, Chan CB, Huang J, Xue S, Yang JJ, et al. Biochemical and biophysical investigation of the brain-derived neurotrophic factor mimetic 7,8dihydroxyflavone in the binding and activation of the TrkB receptor. J Biol Chem. 2014;289:27571-84.

20. Huang EJ, Reichardt LF. Neurotrophins: roles in neuronal development and function. Annu Rev Neurosci. 2001;24:677-736.

21. Massaquoi M, Liguore W, Churchill M, Moore C, Melrose H, Meshul CK. Gait deficits and loss of striatal tyrosine hydroxlase/Trk-B are restored following 7,8dihydroxyflavone treatment in a progressive MPTP mouse model of Parkinson's disease. Neuroscience. 2020;433:53-71.

22. Nie $S$, Ma K, Sun $M$, Lee $M$, Tan $Y$, Chen $G$, et al. 7,8-Dihydroxyflavone protects nigrostriatal dopaminergic neurons from rotenone-induced neurotoxicity in rodents. Parkinson's Dis. 2019;2019:9193534.

23. Li X-H, Dai C-F, Chen L, Zhou W-T, Han H-L, Dong Z-F. 7,8-dihydroxyflavone ameliorates motor deficits Via Suppressinga-synuclein expression and oxidative stress in the MPTP-induced mouse model of parkinson's disease. CNS Neurosci Ther. 2016;22:617-24.

24. Jin H, Zhu Y, Li Y, Ding X, Ma W, Han X, et al. BDNF-mediated mitophagy alleviates high-glucose-induced brain microvascular endothelial cell injury. Apoptosis. 2019:24:511-28.

25. Liu HY, Wei HJ, Wu L, Liu SM, Tang YY, Zou W, et al. BDNF-TrkB pathway mediates antidepressant-like roles of $\mathrm{H}(2) \mathrm{S}$ in diabetic rats via promoting hippocampal autophagy. Clin Exp Pharmacol Physiol. 2020;47:302-12.

26. Al-Bari MAA, Xu P. Molecular regulation of autophagy machinery by mTORdependent and -independent pathways. Ann N Y Acad Sci. 2020;1467:3-20.

27. Corcelle E, Djerbi N, Mari M, Nebout M, Fiorini C, Fénichel P, et al. Control of the autophagy maturation step by the MAPK ERK and p38: lessons from environmental carcinogens. Autophagy. 2007;3:57-59.

28. Moloudizargari $M$, Asghari $\mathrm{MH}$, Ghobadi $\mathrm{E}$, Fallah $\mathrm{M}$, Rasouli $\mathrm{S}$, Abdollahi $\mathrm{M}$. Autophagy, its mechanisms and regulation: Implications in neurodegenerative diseases. Ageing Res Rev. 2017:40:64-74.

29. Yin Y, Sun G, Li E, Kiselyov K, Sun D. ER stress and impaired autophagy flux in neuronal degeneration and brain injury. Ageing Res Rev. 2017;34:3-14.

30. Johnson M, Salvatore M, Maiolo S, Bobrovskaya L. Tyrosine hydroxylase as a sentinel for central and peripheral tissue responses in Parkinson's progression: Evidence from clinical studies and neurotoxin models. Prog Neurobiol. 2018;165-167: 1-25.

31. Klionsky DJ, Abdelmohsen K, Abe A, Abedin MJ, Abeliovich H, Acevedo Arozena $A$, et al. Guidelines for the use and interpretation of assays for monitoring autophagy (3rd edition). Autophagy. 2016;12:1-222.

32. Pasquier B.Autophagy inhibitors. Cell Mol Life Sci. 2016;73:985-1001.

33. Gómez-Santos C, Ferrer I, Reiriz J, Viñals F, Barrachina M, Ambrosio S. MPP+ increases alpha-synuclein expression and ERK/MAP-kinase phosphorylation in human neuroblastoma SH-SY5Y cells. Brain Res. 2002;935:32-39.

34. Chung S, Nagaraju G, Nagalingam A, Muniraj N, Kuppusamy $P$, Walker A, et al. ADIPOQ/adiponectin induces cytotoxic autophagy in breast cancer cells through STK11/LKB1-mediated activation of the AMPK-ULK1 axis. Autophagy. 2017;13:1386-403.

35. Hardie DG. New roles for the LKB1->AMPK pathway. Curr Opin Cell Biol. 2005; 17:167-73.

36. Willows R, Sanders M, Xiao B, Patel B, Martin S, Read J, et al. Phosphorylation of AMPK by upstream kinases is required for activity in mammalian cells. Biochem J. 2017;474:3059-73.

37. Choi JW, Lee J, Park Y.7,8-Dihydroxyflavone attenuates TNF-a-induced skin aging in Hs68 human dermal fibroblast cells via down-regulation of the MAPKs/Akt signaling pathways. Biomed Pharmacother. 2017;95:1580-7.

38. Park H, Park C, Hwang H, Kim B, Kim G, Kim C, et al. 7,8-Dihydroxyflavone attenuates the release of pro-inflammatory mediators and cytokines in lipopolysaccharide-stimulated BV2 microglial cells through the suppression of the NF-KB and MAPK signaling pathways. Int J Mol Med. 2014;33:1027-34.

39. Menzies FM, Fleming A, Caricasole A, Bento CF, Andrews SP, Ashkenazi A, et al. Autophagy and neurodegeneration: pathogenic mechanisms and therapeutic opportunities. Neuron. 2017;93:1015-34.

40. Amin N, Xie S, Tan X, Chen Y, Ren Q, Botchway B, et al. Optimized integration of fluoxetine and 7, 8-dihydroxyflavone as an efficient therapy for reversing depressive-like behavior in mice during the perimenopausal period. Prog. Neuropsychopharmacol. Biol. Psychiatry. 2020;101:109939.
41. Zhang L, Ouyang L, Guo Y, Zhang J, Liu B. UNC-51-like kinase 1: from an autophagic initiator to multifunctional drug target. J Med Chem. 2018;61:6491-6500.

42. Kim J, Kundu M, Viollet B, Guan KL. AMPK and mTOR regulate autophagy through direct phosphorylation of Ulk1. Nat Cell Biol. 2011;13:132-41.

43. Zheng B, Jeong JH, Asara JM, Yuan Y-Y, Granter SR, Chin L, et al. Oncogenic B-RAF negatively regulates the tumor suppressor LKB1 to promote melanoma cell proliferation. Mol Cell. 2009;33:237-47.

44. Zhao J, Du J, Pan Y, Chen T, Zhao L, Zhu Y, et al. Activation of cardiac TrkB receptor by its small molecular agonist 7,8-dihydroxyflavone inhibits doxorubicin-induced cardiotoxicity via enhancing mitochondrial oxidative phosphorylation. Free Radic Biol Med. 2019;130:557-67.

45. Yang $P$, Leu D, Ye K, Srinivasan C, Fike JR, Huang TT. Cognitive impairments following cranial irradiation can be mitigated by treatment with a tropomyosin receptor kinase B agonist. Exp Neurol. 2016;279:178-86.

46. Ryu MJ, Kang KA, Piao MJ, Kim KC, Zheng J, Yao CW, et al. 7,8-Dihydroxyflavone protects human keratinocytes against oxidative stress-induced cell damage via the ERK and PI3K/Akt-mediated Nrf2/HO-1 signaling pathways. Int J Mol Med. 2014;33:964-70.

47. Chen S-D, Wu C-L, Hwang W-C, Yang D-I. More insight into BDNF against neurodegeneration: anti-apoptosis, anti-oxidation, and suppression of autophagy. Int J Mol Sci. 2017:18:545.

48. Nikoletopoulou V, Sidiropoulou K, Kallergi E, Dalezios Y, Tavernarakis N. Modulation of autophagy by BDNF underlies synaptic plasticity. Cell Metab. 2017;26:230-242.e5.

49. Ogawa $N$, Hirose $Y$, Ohara $S$, Ono $T$, Watanabe $Y$. A simple quantitative bradykinesia test in MPTP-treated mice. Res Commun Chem Pathol Pharmacol. 1985;50:435-41.

\section{ACKNOWLEDGEMENTS}

We thank all other members in the Dong laboratories for the helpful discussions and support.

\section{AUTHOR CONTRIBUTIONS}

$L Z$ and $Z D$ conceived the study. $L Z$ and $Z D$ wrote the manuscript. $L Z, C D$, and $L Y$ performed behavioral studies. LZ performed biochemical assays.

\section{FUNDING INFORMATION}

This work was supported by grants from the National Natural Science Foundation of China (NSFC) 82071395,91749116 , and 81622015 , the Natural Science Foundation of Chongqing cstc2020jcyj-zdxmX0004, the Science and Technology Research Program of Chongqing Municipal Education Commission KJZD-K201900403 and Innovation Research Group at Institutions of Higher Education in Chongqing CXQTP19034.

\section{COMPETING INTERESTS}

The authors declare no competing interests.

\section{ETHICS STATEMENT}

All animal experiments were conducted in accordance with the Chongqing Science and Technology Commission guidelines and approved by the Chongqing Medical University Animal Care Committee.

\section{ADDITIONAL INFORMATION}

Supplementary information The online version contains supplementary material available at https://doi.org/10.1038/s41420-021-00643-5.

Correspondence and requests for materials should be addressed to Zhifang Dong.

Reprints and permission information is available at http://www.nature.com/ reprints

Publisher's note Springer Nature remains neutral with regard to jurisdictional claims in published maps and institutional affiliations. 
Open Access This article is licensed under a Creative Commons At c) Atribution 4.0 International License, which permits use, sharing,
adaptation, distribution and reproduction in any medium or format, as long as you give appropriate credit to the original author(s) and the source, provide a link to the Creative Commons license, and indicate if changes were made. The images or other third party material in this article are included in the article's Creative Commons license, unless indicated otherwise in a credit line to the material. If material is not included in the article's Creative Commons license and your intended use is not permitted by statutory regulation or exceeds the permitted use, you will need to obtain permission directly from the copyright holder. To view a copy of this license, visit http://creativecommons. org/licenses/by/4.0/.

(c) The Author(s) 2021 